\title{
Cutaneous immunity and predisposition to infections in diabetic foot population
}

\author{
Pavelina Mihaela Andrei ${ }^{1}$, Maria Daniela Tanasescu ${ }^{1,2}$, Alexandru Minca ${ }^{1,2}$, \\ Andra-Elena Balcangiu-Stroescu ${ }^{3,4}$, Daniela Balan ${ }^{3}$, Andrada Mihai ${ }^{1,5}$, \\ Alexandra Maria Limbau', Constantin Ionescu Tirgoviste ${ }^{1,5}$ \\ ${ }^{1}$ Faculty of Medicine, "Carol Davila" University of Medicine and Pharmacy, Bucharest, Romania \\ ${ }^{2}$ Department of Internal Medicine, University Emergency Hospital, Bucharest, Romania \\ ${ }^{3}$ Faculty of Dental Medicine, "Carol Davila" University of Medicine and Pharmacy, Bucharest, Romania \\ ${ }^{4}$ Department of Dialysis, Emergency University Hospital, Bucharest, Romania \\ ${ }^{5}$ "N.C. Paulescu“ National Institute of Diabetes, Nutrition and Metabolic Diseases, Bucharest, Romania
}

\begin{abstract}
It is unanimously accepted that patients with diabetes have a higher likelihood for infections. The metabolic imbalance associated with diabetes suppresses the immunity system of the cutaneous organ. Hyperglycemia causes an increase of the activity of the sorbitol route with consumption of NADPH. Furthermore, the sorbitol from the cells leads to increase of osmolality in cells as well as reduction of intracellular myo-inositol. These strains on the cellular metabolism expose the cells to oxidative stress. An additional factor is the reduced chemotaxis and phagocytosis impact on the bacterial activity of leukocytes which leads to further predisposition of infection for diabetes patients. Hence the poor control of glycemic levels leads to immunity changes which affects the local immune response against the aggression of pathogenic agents.
\end{abstract}

Keywords: hyperglycemia, immune response, infections

\section{INTRODUCTION}

Diabetes causes a metabolic imbalance which has the root cause chronic hyperglycemia. The chronic hyperglycemic status alters the immune function of the tegument of diabetes patients and the subsiding of the local immune response raises the risk of local infections (1). For diabetes patients most of the cutaneous infections are localized at foot level. Some of the most frequent infections for the diabetic foot are onychomycosis, tinea pedis, ulcerations, cellulitis and gangrene (2). Among the host factors which lead to predisposition to infections at diabetes patients are hyperglycemia, vascular and neuropathic conditions as well as forming of colonies on the skin of pathogenic agents such as Staphylococcus aureus and the Candida species (3).

The cutaneous immunity response is given by leukocytes, mastocytes, monocytes, macrophages, antimicrobial peptides, keratinocytes which produce numerous cytokines such as IL-1, IL-7, IL-7, IL- 8 or growth factors such as TGF- $\alpha$, TGF- $\beta$. Activation of the immune response is the first line of defense against the invasion of pathogenic agents which lead to infections. The appearance of inflammation and the cutaneous signs of it (tumor, calor, dolor, rubor) are attributes of the host immunity system (4).

\section{SUSCEPTIBILITY TOWARDS INFECTIONS GIVEN BY THE HOST FACTORS}

Hyperglycemia reduces the chemotaxis, vascular endothelial adhesion, cellular phagocytosis and the leukocytes bactericidal capacity (5). It causes the intracellular accumulation of sorbitol and fructose which leads to intracellular osmosis resulting in cellular disfunction (6). 
Due to tissue ischemia the vascular function is affected which stimulates the increase in anaerobic organisms and reduced bactericidal ability of leukocytes (5). Vascular permeability is also increased due to the reduction in heparan sulphate and proteoglycans. Furthermore, as the diabetes duration increase, the capillary blood flux is reduced. These alterations result in a slow down of the tissue repair process. Vascular diseases reduce the local inflammatory response and antibiotics absorption which further hinders the healing process $(7,8)$.

Another risk factor which increases the susceptibility of infections is the diabetic sensorial neuropathy. Local minor injuries at foot level caused by accidents caused by the reduced cutaneous sensibility predispose the appearance of ulcers with potential unfavorable evolution $(9,10)$.

The immunologic responses are diminished for diabetic patients. The frequency of onychomycosis and fungal infections as well as secondary bacterial infections is higher compared to that of subjects without diabetes.

\section{THE ROLE OF THE CUTANEOUS MICROBIOME}

The cutaneous microbiome is a complex of commensal and pathogenic organisms. When there is an imbalance in the composition, diversity or metabolites of the microbiome the result is a dysbiosis (11). The bacteria are incapable of penetrating the skin layer without damaging the tissue. Maceration, occlusion, dysbiosis, systemic antibiotherapy lead to change of the $\mathrm{pH}$ level and increase of the bacterial flora (12).

The epidermal lipids have antibacterial proprieties. Free fatty acids and linoleic acid are inhibitors for $S$. aureus. Activated keratocytes produce antimicrobial peptides (AMPs). These are small amphipathic proteins, the cationic side being capable of bonding to microbial membranes while the hydrophobic part allows insertion inside the bacterial lipidic membrane, the final result being the death of the microbe. The most known AMPs from skin level are the cathelicidins (LL-37) and $\beta$ defensins. Among their proprieties are antimicrobial activity done through neutralization of lipopolysaccharides, angiogenesis and contribution to the tissue repairing process (13).

The dysbiosis represents the alteration of the microbiome hemostasis due to systemic changes caused by systemic antibiotherapy of chronic hyperglycemia which leads to immunity suppression or affects external factors such as change in $\mathrm{pH}$ levels or heightened temperature, hence non-pathogen germens commensals in physiological conditions can lead to serious infections $(14,15)$.

\section{CUTANEOUS INFECTIONS}

Diabetic foot infections are associated with an increased morbidity and mortality due to the aggressive nature of the phenomena as well as the collapse of the immune system (16). Among the risk factors which make the patient susceptible to these infections are the peripheral neuropathy, peripheral vascular disease, local injuries and the chronical hyperglycemic status. The reduction of the local pain and temperature stimuli slow down the perception of local lesions at foot level. Reduction in glandular secretion as a result of autonomous neuropathy leads to cutaneous xerosis as well as pruritus and local fissures which represents an entry gateway for microorganisms (17). Foot deformation caused by motoric neuropathy favors microtraumas creation due to improper position of plantar pressure during walking. Tissue which suffer from ischemia and hypooxigenation are a favoring factor for the proliferation of anaerobic microorganisms (18).

Dermatoses caused by mycotic agents manifests itself predominantly in the intertriginous areas and at nails level. These regions have high humidity. Tinea pedis and onychomycosis are the most frequent mycotic infections, the pathogen agents being Trichophyton rubrum and Candida albicans. The clinical presentation is in the form of soft squamous erythematous plaques or patches located in the interdigital or plantar regions. Yellow or white patches found on the distal and lateral edge of the nail, with onychodystrophy and hyperkeratosis are linked to onychomycosis. Dermatophytosis is frequently encountered at diabetes patients (19).

Bacterial infections due to positive gram aerobic cocci such as Staphylococcus aureus, Streptococcus agalactiae, Streptococcus pyogenes and coagulase-negative staphylococci are the most frequent causes for cellulitis and ulcers with secondary infections. Clinically these are profound, most often treated with systemic antibiotic therapy, which in time develops to methicillin resistant strains (MRSA). Enabling of various microbial agents favors the progression towards abscesses, gangrene or septicemia. The hyaluronidase produced by the streptococcus facilitates the spread of the necrotizing toxins of the staphylococcus. Furthermore, these pathogenic agents produce enzymes with an- 
giotoxic effects which favor the appearance of thrombosis in situ thus compromising local circulation. Cellulitis and abscess complicate the superinfected ulcers. These involve the deep derma with collections of puss and signs of local and systemic inflammation. These infections can extend themselves even further, reaching the bone or the systemic circulation (20).

The pathogenic factors of Staphylococcus aureus are represented by the surface antigens, extracellular enzymes and exotoxins. The attachment of the staphylococcus in the tissues is done through the teichoic acids, the peptidoglycan which stimulates the secretion of cytokines responsible for the state of shock and the A protein which fixes the Fc of IgG thus preventing opsonization and phagocytosis (21). Extracellular enzymes are at the base of tissue lesions while also producing pathogen agent dissemination. These are represented by free coagulase which in turn determines the transformation of fibrinogen into fibrine and coagulation of the plasma responsible of sceptic thrombophlebitis and bound coagulase which is antiphagocytic. The staphylokinase (SAK, fibrinolysin) inactivates the defensins from skin level, activates the plasminogen from plasmin and it's also responsible for bacterial dissemination. Surface plasmin cleaves $\mathrm{C} 3 \mathrm{~b}$ and $\mathrm{G}$ immunoglobulin eliminating the opsonization from the bacteria surface. The inhibition protein of the chemotaxis bounds to C5a blocking the activity of neutrophiles. The inhibitor of the staphylococcic complement bounds to $\mathrm{C} 3$ convertase on the surface of the bacteria preventing the $\mathrm{C} 3$ splitting and activating the complement's cascade (22). Exfoliative toxins, exfoliatin A and exfolatin B, have cutaneous trophism, enterotoxins (A-C) and toxic shock syndrome toxin 1 (TSST 1). Exfoliants $\mathrm{A}$ and $\mathrm{B}$ cleave desmoglein 1 from the superficial epidermal level leading to loss of intracellular cohesion, the epidermolysis taking place in the granulated layer, with the final result being a flaccid bubble with positive Nikolsky sign (4).

As a virulent mechanism for GAS infections, these have the capacity to inactivate the cathelicidins (LL-37) applying resistance to the inherited immune system. Streptococcal pyrogenic exotoxins A and C secreted by the Streptococcus pyogenes are capable of system invasion.

An infection of soft tissue with vital risk is necrotizing fasciitis. It's most often caused by anaerobic species Bacteroides, Clostridium, Peptostreptococcus, Enterobacter, Klebsiella, Proteus and Streptococcus anaerobius group A (GAS). The infection can be polymicrobial or monomicrobial (more frequently for GAS or less frequently for Staphylococcus aureus). Increase in severity is fast. Complications such as sepsis or multiple organ failure are quickly to happen if a prompt intervention is not done. Streptococcus pyogenes (GAS) contains $\mathrm{M}$ protein which has high virulence due to its antiphagocytic effect (21).

\section{KEY STEPS}

Evaluation of a suspicion of infection of the diabetic foot seeks the identification of favorable factors for infection, microbial etiology evaluation as well as determining the severity and expansion of the infection. Clinical history, recent traumas, the length and evolution of the infection or mechanical factors which predispose the infection development, together with glycemic control and systemic symptoms will be evaluated, classified and treated according to the severity level they represent.

Clinical evaluation must also include neurological examination which shows the presence of loss of perception or stimuli as well as vascular examination for evaluation of vascular disease presence (23).

Laboratory paraclinical parameters, cultivation from secretion and bone radiography allows to evaluate the spread of the infection.

Enhancing the glycemic control reduces the risk of complications both microvascular and macrovascular as well as infections at diabetes patients.

The infections are more frequent at patients diagnosed with diabetes than for subjects without diabetes. The infections can lead to metabolic decompensation which can go as far as ketoacidosis or hyperosmolar comas. Foot gangrene represents the most sever infection which comes with a high risk of amputation $(24,25)$. The defense capacity of the diabetic patient is severely compromised hence the chemotaxis, vessel wall adhesion and leukocyte phagocytic function are reduced. Monocytes alteration lead to a reduced detection capacity of pathogenic agents. The high proneness to infection of diabetic patients is also caused by the fall of cellular mediated immunity due to reduced production of interleukin 2 and due to the reduction of the response of cellular natural killer (NK) to interferon. The diabetic neuropathy and vascular affection compromise the tissue trophicity as well as the regeneration potential.

\section{CONCLUSIONS}

There is a bidirectionality between the infection and the diabetes since the diabetes favors the infec- 
tion while the infection creates a prolonged metabolic imbalance. A simultaneous approach of the infection, the diabetes and the risk factor will prevent the metabolic decompensation and the complications which result from it.

\section{REFERENCES}

1. Demirseren DD, Emre S, Akoglu G, Arpacı D, Arman A, Metin A et al. Relationship between skin diseases and extracutaneous complications of diabetes mellitus: Clinical analysis of 750 patients. Am J Clin Dermatol. 2014;15(1):65-70.

2. Lima AL, Illing T, Schliemann S, Elsner P. Cutaneous Manifestations of Diabetes Mellitus: A Review. Am J Clin Dermatol. 2017;18(4):54153

3. De Macedo GMC, Nunes S, Barreto T. Skin disorders in diabetes mellitus: An epidemiology and physiopathology review. Diabetol Metab Syndr. 2016;8(1):1-8.

4. Lowell A. Goldsmith, Stephen I. Katz, Barbara A. Gilchrest, Amy S. Paller, David J. Leffell KW. Fitzpatrick's Dermatology in General Medicine, Eighth Edition. 2012.

5. Delamaire M, Maugendre D, Moreno M, Le Goff MC, Allannic H, Genetet B. Impaired leucocyte functions in diabetic patients. Diabet Med. 1997;14(1):29-34 .

6. Malgrange D. Physiopathology of the diabetic foot [French Physiopathologie du pied diabetique]. Rev Med Interne. 2008;29(Suppl. 2):S231-7.

7. Beks PJ, Mackaay AJC, Neeling JND De, Vries H De, Bouter LM, Heine RJ. Peripheral arterial disease in relation to glycaemic level in an elderly Caucasian population: The Hoorn Study. Diabetologia. 1995;38:86-96.

8. Marso SP, Hiatt WR. Peripheral arterial disease in patients with diabetes. J Am Coll Cardiol. 2006;47(5):921-9.

9. Tesfaye S, Stevens LK, Stephenson JM, Fuller JH, Plater M, Ionescu-Tirgoviste $\mathrm{C}$ et al. Prevalence of diabetic peripheral neuropathy and its relation to glycaemic control and potential risk factors: The EURODIAB IDDM Complications Study. Diabetologia. 1996;39(11):1377-84.

10. Singh R, Barden A, Mori T, Beilin L. Advanced glycation end-products: A review. Diabetologia. 2001;44(2):129-46.

11. Dreno B, Araviiskaia E, Beradesca E, Gontijo G, Sanchez M, Xiang LF et al. Microbiome in healthy skin, update for dermatologists. J Eur Acad Dermatology Venerol. 2016;1-10.

12. Mirela SL, Gabriela C, Mihail A. The skin's microbiota and microbiome. Rev Soc Rom Dermatologie. 2017;83-91.

\section{Acknowledgement}

All authors contributed equally to the present work and thus are main authors.

Conflict of interest: none declared Financial support: none declared

13. Xia X, Cheng L, Zhang S, Wang L, Hu J. The Role of Natural Antimicrobial Peptides During Infection and Chronic Inflammation. Antonie Van Leeuwenhoek. 2018 Jan;111(1):5-26.

14. Human T, Project M. Structure, function and diversity of the healthy human microbiome. Nature. 2012;486(7402):207-14.

15. Gardiner M, Vicaretti M, Sparks J, Bansal S, Bush S, Liu M et al. A longitudinal study of the diabetic skin and wound microbiome. Peer J. 2017;5:e3543.

16. Singh N, Armstrong DG, Lipsky BA. Preventing foot ulcers. J Am Med Assoc. 2005;293(2):94-6.

17. Hokkam EN. Assessment of risk factors in diabetic foot ulceration and their impact on the outcome of the disease. Prim Care Diabetes. 2009;3(4):219-24.

18. Creager MA, Lüscher TF, Cosentino F BJ. Diabetes and vascular diseases.Pathophysiology, Clinical Consequences, and Medical Therapy: Part I. Circ J Am Hear Assoc. 2003;108:1527-32.

19. Pătrașcu V. Boli dermatologice și infecții sexual-transmisibile. Ed. Sitech, 2018.

20. Lipsky BA, Senneville E, Abbas ZG, Aragón-Sánchez J, Diggle M et al. on behalf of the International Working Group on the Diabetic Foot (IWGDF). IWGDF Guideline on the diagnosis and treatment of foot infection in persons with diabetes. 2019. Available from: https:// iwgdfguidelines.org/wp-content/uploads/2019/05/05-IWGDF-infectionguideline-2019.pdf.

21. Chastain CA, Klopfenstein N, Serezani CH, Aronoff DM. A Clinical Review of Diabetic Foot Infections. Clin Podiatr Med Surg. 2019;36(3):381-95.

22. Bartelink ML, Hoek L, Freriks JP, Rutten GEHM. Infections in patients with type 2 diabetes in general practice. Diabetes Res Clin Pract. 1998;40:15-9.

23. Bateman S. Principles of preventative foot care. Br J Community Nurs. 2014;\$30-8.

24. Pecoraro RE, Reiber GE, Burgess EM. Pathways to diabetic limb amputation: Basis for prevention. Diabetes Care. 1990;13(5):513-21.

25. Sen P, Demirdal T, Emir B. Meta-analysis of risk factors for amputation in diabetic foot infections. Diabetes Metab Res Rev. 2019;35(7):e3165. 